\title{
Anlotinib Suppresses Colorectal Cancer Proliferation and Angiogenesis via Inhibition of AKT/ERK Signaling Cascade
}

This article was published in the following Dove Press journal: Cancer Management and Research

\author{
Qian Yang ${ }^{1, *}$ \\ Laichao $\mathrm{Ni} \mathbb{D}^{1, *}$ \\ Saber Imani ${ }^{1, *}$ \\ Zhangqiang Xiang' \\ Rui Hai ${ }^{1}$ \\ Ruilin Ding' \\ Shaozhi Fu (D) \\ Jing bo $\mathrm{Wu}^{\mathrm{l}, 2}$ \\ Qinglian Wen'

\begin{abstract}
'Department of Oncology, The Affiliated Hospital of Southwest Medical University, Luzhou, Sichuan 646000, People's

Republic of China; ${ }^{2}$ Nuclear Medicine and Molecular Imaging Key Laboratory of Sichuan Province, Department of Nuclear Medicine, The Affiliated Hospital of Southwest Medical University, Luzhou, Sichuan 646000, People's Republic of China

*These authors contributed equally to this work
\end{abstract}

Background: Anlotinib is a highly potent multi-target tyrosine kinase inhibitor, with very good anti-tumor activity against a variety of solid tumors. However, its effect on colorectal cancer (CRC) is not yet clearly understood. The objective of this study was to investigate the anti-tumor effect and underlying mechanism of anlotinib in the pathogenesis of CRC.

Materials and Methods: Effects of anlotinib on CT26 cells proliferation and microvessel formation in endothelial cells were determined by MTT assay and tube formation assay. Cell migration and invasion were analyzed by using the wound healing assay and transwell assay. Cell cycle and apoptosis were detected by flow cytometry. A CRC xenograft mouse model was used for conducting in-vivo studies to verify the effect of anlotinib. The expression of Ki-67 and CD31 in the tumor tissue was detected by immunohistochemistry and protein expression was measured by Western blot.

Results: In-vitro studies revealed that anlotinib inhibited the proliferation, migration, and invasion of CT26 cells and the tube formation of HUVECs in a dose-dependent manner. Anlotinib also significantly induced cell apoptosis and G2/M arrest. It effectively inhibited tumor growth and prolonged survival time in the CRC xenograft mouse model. Immunohistochemical analysis of the tumor tissue revealed that anlotinib downregulated CD31 and Ki-67 which are the biomarkers of microvessel density and proliferation. Furthermore, anlotinib was able to inhibit the activation of VEGFR-2/AKT and FGFR, PDGFR $\beta$ and their downstream signaling ERK.

Conclusion: The findings of the present study suggested that anlotinib suppressed cell proliferation and angiogenesis via inhibition of AKT/ERK signaling pathway in colorectal cancer and could be a novel therapeutic strategy for treatment of CRC.

Keywords: anlotinib, colorectal cancer, angiogenesis, proliferation, tyrosine kinase inhibitor

\section{Introduction}

Colorectal cancer (CRC) is the third most prevalent malignancy of the gastrointestinal tract. Over 1.8 million new cases of CRC were reported worldwide in 2018. CRC continues to be the second leading cause of cancer-associated mortality, contributing to approximately 900,000 associated deaths each year. ${ }^{1,2}$ Surgical resection is the cornerstone for the treatment of CRC. Postoperative adjuvant chemotherapy is routinely recommended to reduce the recurrence and improve prognosis in CRC patients. However, approximately $50 \%$ of the patients suffering from CRC develop recurrence and distant metastasis (mCRC)., Targeted cancer therapies have attracted considerable attention in recent times.
Correspondence: Qinglian Wen Department of Oncology, The Affiliated Hospital of Southwest Medical University, Southwest Medical University, 3-319

Zhongshan Road, Luzhou, Sichuan 646000, People's Republic of China $\mathrm{Tel} / \mathrm{Fax}+86-830-3160283$

Emailwq173II5@hotmail.com 
The emergence of molecular targeted therapies has prolonged the overall survival (OS) of patients with mCRC from 6-12 months to nearly 30 months. ${ }^{5}$ Although the introduction of molecular targeted drugs has significantly improved the prognosis of patients with $\mathrm{mCRC}$, due to the heterogeneous nature of cancer, primary resistance caused by lacks of gene mutations or secondary resistance after using drugs have made some patients with poor treatment or disease progression. ${ }^{6}$ Thus, prognosis of CRC remains unsatisfactory due to the development distant metastasis and limitations of current therapies for management of mCRC. Therefore, there is an urgent need for development of novel and effective drugs and therapeutic strategies for management of patients with metastatic CRC. ${ }^{7}$

Anlotinib, a novel orally administered potent multitargeted receptor tyrosine kinase inhibitor that targets vascular endothelial growth factor receptor (VEGFR), platelet-derived growth factor receptor (PDGFR), fibroblast growth factor receptor (FGFR), and stem cell factor receptor (c-Kit), has been developed independently in China. ${ }^{8}$ Multiple studies have tumor cell growth, proliferation, angiogenesis, and metastasis in several human cancers, including non-small cell lung cancer (NSCLC), ${ }^{9}$ hepatocellular carcinoma (HCC), ${ }^{10}$ osteosarcoma, ${ }^{11}$ and thyroid cancer ${ }^{12}$ can be effectively suppressed by anlotinib. VEGF triggered a network of downstream signaling pathways to promote tumor angiogenesis, metastasis, and growth by activating three structurally related VEGF receptor tyrosine kinases including VEGFR1, VEGFR2, and VEGFR3 ${ }^{13-18}$ Notably, anlotinib markedly inhibited the activation of VEGFR2, VEGFR3, PDGFR $\beta$, and FGFR1. Thus, anlotinib exerted an inhibitory effect on angiogenesis induced by VEGF, PDGF, and FGF-2 and may serve as a potential therapeutic agent for inhibition of angiogenesis in tumors. ${ }^{8,19}$

Recently, Phase II and III clinical trials demonstrated that anlotinib exhibited noticeable anti-tumor activity in a variety of solid tumors, including NSCLC, renal cancer, thyroid medullary cancer and soft tissue sarcoma. Anlotinib was approved by the China Food and Drug Administration (CFDA) as a third-line treatment for patients with advanced NSCLC in 2018. ${ }^{20-22}$ Anlotinib therapy may however exhibit different clinical response in different cancers due to heterogeneity of tumors. Besides, the antineoplastic activity and potential anti-tumor effect of anlotinib in CRC has not been characterized.

Therefore, the objective of this study was to investigate the therapeutic effect of anlotinib on CRC and to understand the probable underlying molecular mechanisms and signaling pathways via which it exerted an anti-tumor effect. Herein, we found that anlotinib potentially inhibited the activation of VEGFR-2, FGFR, and PDGFR $\beta$. It significantly inhibited cell proliferation, migration, invasion and angiogenesis. The findings of this current study revealed that anlotinib produced a good antitumor effect in CRC by affecting several malignancy endpoints via down-regulation of the AKT/ERK signaling cascade. Overall, this study provided prospective evidence for the clinical application of anlotinib in the therapeutic management of CRC.

\section{Materials and Methods Drugs and Reagents}

Anlotinib was obtained from Chia Tai Tianqing pharmaceutical group (Tianqing Co., Nanjing, Jiangsu, China). The antibodies against p-VEGFR2, p-FGFR, and p-PDGFR $\beta$ were procured from Cell Signaling Technology (Bioss Co., Beijing, China). Antibodies against VEGFR2, FGFR, PDGFR $\beta$, ERK1/2, p-ERK1/2, AKT, and p-AKT were purchased from Cell Signaling Technology (Servicebio Co., Wuhan, China).

\section{Cell Line and Cell Culture}

The murine colorectal carcinoma cell line (CT26 cells) and Human umbilical vein endothelial cell line (HUVECs) were purchased from American Type Culture Collection (ATCC, Manassas, VA, USA). The CT26 cells and HUVECs were cultured in Dulbecco's Modified Eagle's medium (DMEM) and Roswell Park Memorial Institute (RPMI) 1640 medium supplemented with $10 \%$ fetal bovine serum (Thermo Fisher Scientific, Waltham, MA, USA) and $1 \%(\mathrm{v} / \mathrm{v})$ antibiotics (100 units $/ \mathrm{mL}$ penicillin and $100 \mu \mathrm{g} / \mathrm{mL}$ streptomycin; Sigma-Aldrich, St Louis, MO, USA) in a humidified atmosphere of $5 \% \mathrm{CO}_{2}$ at $37{ }^{\circ} \mathrm{C}$.

\section{Cell Viability Assay}

Cell proliferation activity was determined using the standard MTT assay (Abcam, Shanghai, China), following the manufacturer's instructions. Briefly, CT26 cells were seeded into 96-well plates at a density of 5000 cells/well. After the cells were treated with different concentrations of anlotinib $(0,0.25,0.5,1,2,4,8,16,32 \mu \mathrm{mol} / \mathrm{L})$ and incubated at $37^{\circ} \mathrm{C}$ for 24 hours, 48 hours, and 72 hours. Subsequently, $20 \mu \mathrm{L}$ of the solution of MTT $(5 \mathrm{mg} / \mathrm{mL})$ was added in each well and the cells were again incubated at $37^{\circ} \mathrm{C}$ for 4 hours. Finally, $150 \mu \mathrm{L}$ of dimethyl sulfoxide 
(DMSO) was added to each well and the absorbance of the solution was measured at $490 \mathrm{~nm}$ on an iMark microplate reader (Bio-Rad, CA, USA).

\section{Cell Migration Assay}

The wound-healing migration assays were performed as described previously. ${ }^{23}$ In brief, CT26 cells were uniformly seeded into a 12 -well plate and were incubated at $37{ }^{\circ} \mathrm{C}$ until they reached about $90 \%$ confluence. A scratch line "wound" was subsequently created in the cell monolayer with a sterile $10-\mu \mathrm{L}$ pipette tip perpendicular to the bottom of the plate and cells were washed three times with phosphate buffered saline (PBS) to remove scraped or dead cells. CT26 cells were then cultured in a serum-free medium containing different concentrations $(0,1,2,4 \mu \mathrm{mol} / \mathrm{L})$ of Anlotinib at $37{ }^{\circ} \mathrm{C}$ for 24 hours. Cell migration was observed under a phase-contrast microscope (Olympus Cor., Tokyo, Japan) at 0-24 h post-induction of injury. The differences in migration were analyzed using ImageJ software with Wound Healing Tool plug-in.

\section{Transwell Invasion Assay}

The invasion assay was carried out using a 24-well transwell chambers with $6.5 \mathrm{~mm}$ diameter polycarbonate filters ( $8 \mu \mathrm{m}$ pore size, Corning, NY, USA) that coated with $35 \mu \mathrm{L}$ Matrigel (Becton, Dickinson and Company, USA). Cells were seeded in the upper compartments at a density of $5 \times 10^{4}$ cells/well and then treated with different concentrations of Anlotinib $(0,2,4,8 \mu \mathrm{mol} / \mathrm{L})$, Culture medium containing $10 \%$ FBS was added to the lower chambers. The plates were incubated for 24 hours at $37^{\circ} \mathrm{C}$. After the chambers were washed twice with PBS, the fixed cells were treated with methanol for $20 \mathrm{~min}$ and stained with Giemsa stain for $15 \mathrm{~min}$. The inner side of the chambers were wiped with a cotton swab. Images were acquired using an inverted microscope (Olympus Cor., Tokyo, Japan), and the invaded cells were counted manually in 5 randomly selected fields.

\section{Tube Formation Assay}

$60 \mu \mathrm{L} /$ well of cold Matrigel (Becton, Dickinson and Company, USA) was added to a prechilled 96-well plate and solidified at $37^{\circ} \mathrm{C}$ for 30 minutes. HUVECs $\left(1 \times 10^{5}\right.$ cells $/ \mathrm{mL}$ ) were cultured in 1640 culture medium containing $10 \% \mathrm{FBS}$ and different concentrations of anlotinib (0, $1,2,4 \mu \mathrm{mol} / \mathrm{L})$ and added to each Matrigel-containing well. The cells were incubated for 6 hours at $37^{\circ} \mathrm{C}$ after which the structure of forming tubes was observed with a microscope (Olympus Cor., Tokyo, Japan).

\section{Cell Cycle and Apoptosis Assays}

Cells were cultured $\left(1 \times 10^{6} / \mathrm{mL}\right)$ for 24 hours with different concentration of Anlotinib (0, 2, 4, $8 \mu \mathrm{mol} / \mathrm{L})$. The treated cells were harvested and washed twice with cold PBS after which they were fixed with 70\% ice-cold ethanol for 12 hours at $4{ }^{\circ} \mathrm{C}$. The cells were then incubated for 30 minutes with a mixture containing $0.5 \mathrm{~mL}$ staining buffer, $25 \mu \mathrm{L}$ propidium iodide staining solution $(20 \mathrm{X})$ and $10 \mu \mathrm{L}$ RNase A (50X). Cell cycle distribution was analyzed using a flow cytometer with PI/RNase staining buffer (BD Biosciences, CA, USA). Cell apoptosis assay was performed using FITC Annexin V Apoptosis Detection standard kit (BD Biosciences, CA, USA). The cells were briefly treated with $0,2,4,8 \mu \mathrm{mol} / \mathrm{L}$ concentrations of Anlotinib for 48 hours. They were then digested and washed twice with cold PBS and then resuspended in $1 \mathrm{X}$ Binding Buffer. Apoptotic and dead cells were detected by flow cytometry after staining with Annexin V-FITC and propidium iodide (PI). The frequencies of $\mathrm{PI}+$ and Annexin V-FITC+ cells were analyzed using FACS Canto II flow cytometer (BD Biosciences, CA, USA); between 500,000-1,000,000 events were assessed in each tube. FlowJo software (version 10.0) was used for analyzing the data.

\section{Xenograft Mouse Model}

The animals were cared for in accordance with the Guide for the care and use of laboratory animals in China. All procedures and animal experiments were approved by the Animal Protection and application committee of Southwest Medical University, Luzhou, Sichuan, China.

Forty-eighty female BALB/c mice (age 4-5 weeks, weight 200 to $250 \mathrm{~g}$ ) were obtained from the Dashuo Animal Laboratory Center (Chengdu, China). The mice were housed under specific-pathogen-free (SPF) controlled conditions of $22 \pm 1{ }^{\circ} \mathrm{C}$ temperature, $60 \% \pm 10$ humidity with a 12-h light/dark cycle and ad libitum access to water and sterile food pellets. The subcutaneous CRC xenograft model was established by administering $100 \mu \mathrm{L}$ suspensions of CT26 cells $\left(1 \times 10^{6}\right.$ cells $\left./ \mathrm{mL}\right)$ into the right flanks of BALB/ c mice. The cells were allowed to grow in the animals for 2 weeks until the tumor size was approximately $200 \mathrm{~mm}^{3}$ in volume. Forty-eighty mice were randomly divided into four groups $(\mathrm{n}=12)$ : Normal saline (NS) group, $0.75 \mathrm{mg} / \mathrm{kg}$ anlotinib group, $1.5 \mathrm{mg} / \mathrm{kg}$ anlotinib group, and $3 \mathrm{mg} / \mathrm{kg}$ anlotinib group and were treated by intragastric administration for 2 weeks. After 21 days, six mice in each group were 
anesthetized with ether and sacrificed. The tumor tissues including the heart, lung, liver, and kidney were dissected carefully and washed with sodium chloride solution and then collected and fixed with $10 \%$ buffered formalin for 24 hours at room temperature after which they were embedded in paraffin. Tumor volumes were calculated every 2 days using the following standard formula: $0.5 *$ length*width $2 .^{24}$ The survival periods were also evaluated (6 mice per group) to further evaluate the anti-tumor effect of anlotinib against CRC.

\section{Histopathology and Immunohistochemistry Assay}

Formalin-fixed tissue samples were embedded in paraffin and sectioned into 4- $\mu \mathrm{m}$-thick sections. The sections from the primary tumor, heart, lung, liver, and kidney were stained with hematoxylin and eosin, and the tumor specimens were immunostained with Ki-67 (Bio-World, CA, USA) and CD31 (Bio-World, CA, USA). To quantify Ki67 and $\mathrm{CD} 31$ expression, the Ki-67 labeling index was calculated in five randomly selected areas in each tumor sample as the number of Ki-67 positive cells/total counted at $400 \times$ magnification. Besides, by calculating the area percentage of positive expression of CD31, five high power visual fields (X400) were randomly selected for each section. The proportion of CD31 positive expression was calculated as the area of CD31 positive staining/the total area under a high magnification field of vision. All these counts were performed in a blinded manner. The sections were visualized with a Zeiss Axioplan 2 microscope, and images were captured on a Windows NT workstation and analyzed using Zeiss Axiovision software (Zeiss; New York, NY).

\section{Western Blotting Analysis}

The expression of AKT/ERK signaling cascade proteins were detected using Western blot analysis as described previously. ${ }^{25}$ In brief, the tissue samples were weighed $(0.5 \mathrm{~g})$, homogenized and centrifuged at $14,000 \mathrm{rpm}$ at 4 ${ }^{\circ} \mathrm{C}$ for $20 \mathrm{~min}$. Supernatant was collected and preserved at $-80^{\circ} \mathrm{C}$. The total proteins were then extracted on ice using a protein extraction buffer supplemented with protease inhibitors cocktail. Protein concentrations were quantified using the BCA Protein Assay Kit (KeyGEN BioTECH). Cell lysates were separated by $10 \%$ sodium dodecyl sulfate-polyacrylamide (SDS-PAGE) gel electrophoresis (SDS-PAGE) and after which the proteins transferred on to polyvinylidene difluoride (PVDF) membrane (Millipore, Corporation, Billerica, MA, USA). The membrane was blocked with $4 \%$ non-fat dry milk in Trisbuffered saline with Tween-20 for 1 hour at $20^{\circ} \mathrm{C}$. Subsequently, the membrane was incubated with primary antibodies against VEGFR2, p-VEGFR2, FGFR, p-FGFR, PDGFR $\beta$, p-PDGFR $\beta$, ERK1/2, p-ERK1/2, AKT, and p-AKT, respectively using a dilution of 1:200 for each antibody. The membrane was incubated overnight at $4{ }^{\circ} \mathrm{C}$ with the respective primary antibody in $5 \%$ blocking buffer.

Then, the membrane was washed three times with PBS and incubated with the goat anti-rabbit secondary antibody (1:3000) (Beyotime Institute of Biotechnology, Shanghai, China) at room temperature for 1 hour. The protein bands were visualized using the enhanced chemiluminescence detection kit (Bio-Rad, CA, USA.) and the protein bands were quantified and analyzed using photo-shop image software.

\section{Statistical Analyses}

Statistical analyses were performed using the SPSS software package (version 20.0; IBM, Chicago, IL, USA). All graphs were produced using Graph Pad Prism 5.0 for Windows software (Graph Pad Software Inc., La Jolla, CA, USA). All experiments were performed in triplicate and the data represents means from three independent experiments. Data are presented as mean \pm standard deviation (SD) or median (range) for variables and were compared using a Student's $t$-test for two groups, or one-way ANOVA for multiple groups. A P-value of $<0.05$ was considered to be statistically significant.

\section{Results}

\section{Anlotinib Inhibits the Cell Proliferation, Migration, Invasion and Angiogenesis in vitro}

The effect of anlotinib on the proliferative activity of CT26 cells and the tube formation of HUVECs were evaluated by using the MTT and tube formation assay. As shown in Figure 1C, the growth of CT26 cells was significantly reduced with increasing concentrations of anlotinib $(0,0.25,0.5,1,2,4,8,16$, and $32 \mu \mathrm{mol} / \mathrm{L} ; P<$ $0.05)$. Moreover, cell growth was also significantly inhibited when the treatment time was prolonged. The IC50 values with 24 hours, 48 hours and 72 hours of anlotinib treatment were $16.16 \mu \mathrm{mol} / \mathrm{L}, 11.73 \mu \mathrm{mol} / \mathrm{L}$, and 2.89 
A

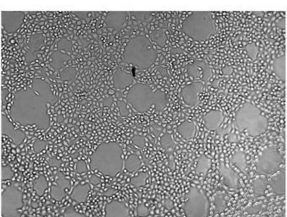

NS

B

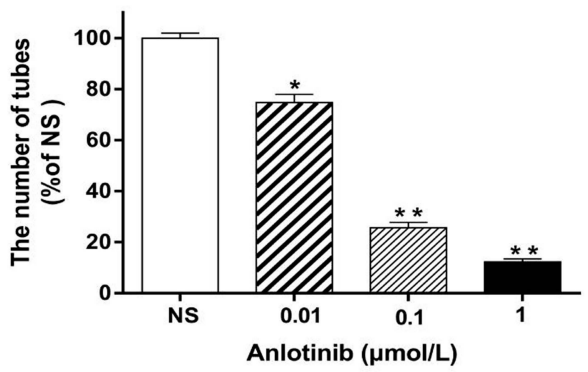

D
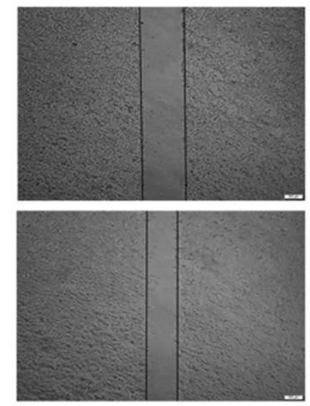

NS

E
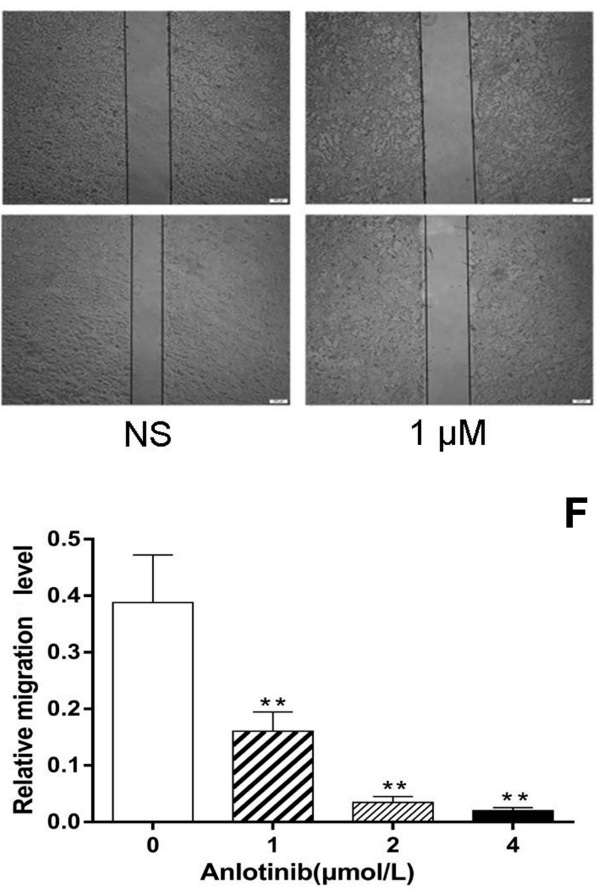

$1 \mu \mathrm{M}$

F

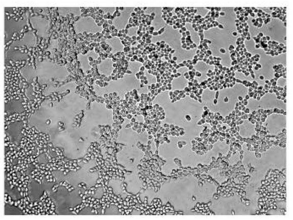

$0.1 \mu \mathrm{M}$

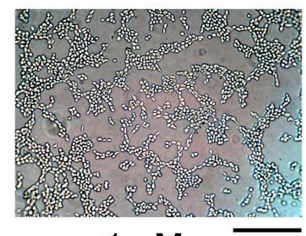

$1 \mu \mathrm{M} \quad \overline{100 \mu \mathrm{m}}$

C
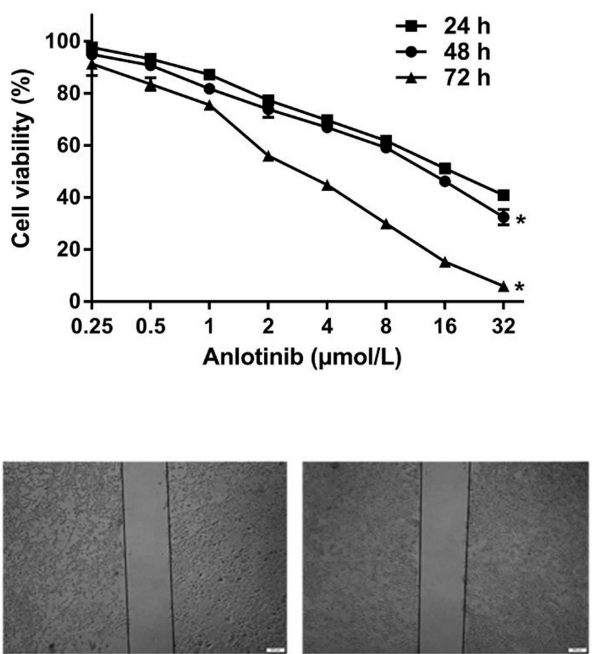

$\mathrm{Oh}$

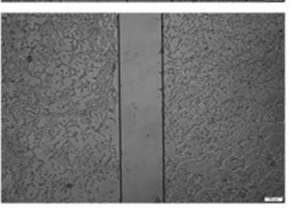

$2 \mu \mathrm{M}$

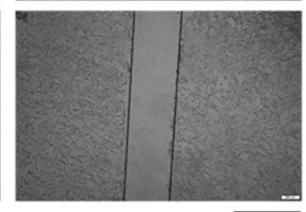

$4 \mu \mathrm{M} \quad \overline{200 \mu \mathrm{m}}$

$24 \mathrm{~h}$

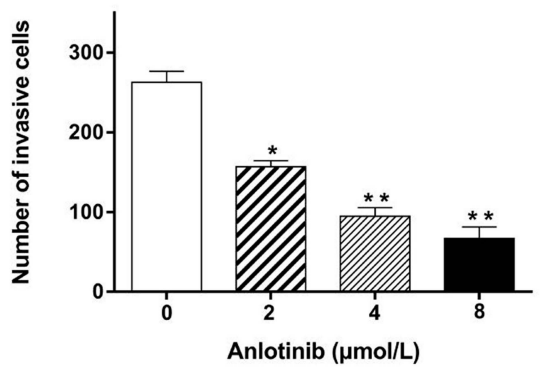

G

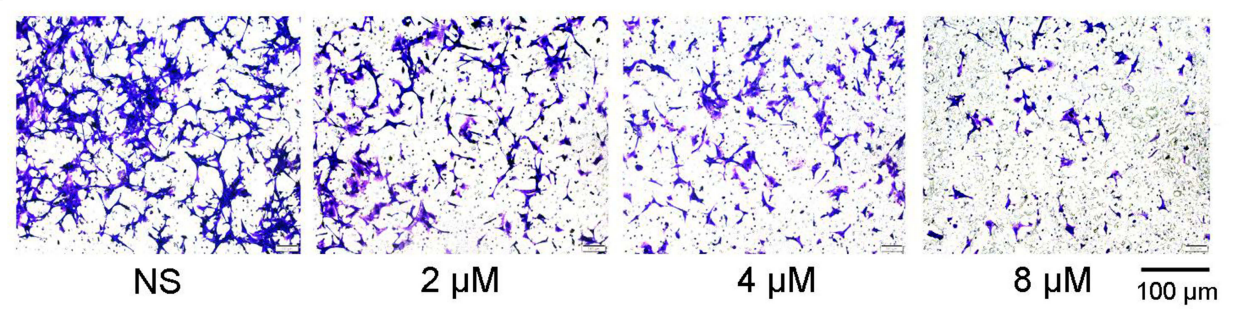

Figure I Anlotinib inhibits cell proliferation, migration, and invasion of CT26 cells and the tube formation of HUVECs. (A) Representative images of the tube formation assay. (B) Number of tube formation with different concentrations of anlotinib as a percentage of NS group. (C) Proliferation activity of cells after incubation for 24, 48, and $72 \mathrm{~h}$ with different concentrations of anlotinib. (D) Representative images of migration at 0 and $24 \mathrm{~h}$ post-induction of injury in a wound-healing assay. (E) Histogram showing relative migration level. (C) Proliferation activity of cells after incubation for 24, 48, and $72 \mathrm{~h}$ with different concentrations of anlotinib. (F) Representative images of a transwell assay. (G) The quantification of invading cell numbers with different concentrations of Anlotinib. Data are presented as mean \pm SD of three independent experiments. ( $* P<0.05$ and $* * P<0.01$ versus NS group). 
$\mu \mathrm{mol} / \mathrm{L}$, respectively. Anlotinib also inhibited the microvessels formation of HUVECs in a concentrationdependent way (Figure 1A). The formation of enclosed tubes were visibly decreased following treatment with 0.1 $\mu \mathrm{mol} / \mathrm{L}$ and $1 \mu \mathrm{mol} / \mathrm{L}$ anlotinib when compared with the NS group $(P<0.01)$ (Figure 1B).

In addition, we also performed wound-healing and transwell assays to investigate whether anlotinib inhibited the CRC cell migration and invasion. The migration capability of CT26 cells progressively decreased with increasing concentrations of Anlotinib (Figure 1D and E). These results clearly indicated that $1 \mu \mathrm{mol} / \mathrm{L}, 2 \mu \mathrm{mol} / \mathrm{L}$, and 4 $\mu \mathrm{mol} / \mathrm{L}$ of anlotinib significantly inhibited cell migration of CT26 cells after $24 \mathrm{~h}$ of treatment when compared with the NS group $(P<0.01)$. Invading cell number at different concentrations of anlotinib has been illustrated in Figure $1 \mathrm{~F}$ and $\mathrm{G}$ ). Interestingly, $4 \mu \mathrm{mol} / \mathrm{L}$ and $8 \mu \mathrm{mol} / \mathrm{L}$ concentrations of anlotinib exerted the highest effect in reducing the number of invading cells when compared with NS group $(95 \pm 11$ cell $/ \mathrm{mL}$ and $67 \pm 14$ cell $/ \mathrm{mL}$, respectively vs. $263 \pm 13$ cell $/ \mathrm{mL}, P<0.01$ for both). Thus, increasing concentrations of anlotinib significantly suppressed the migration of CT26 cells and the number of cells migrating through the Transwell ventricular membrane. Taken together, these results indicated that anlotinib can significantly inhibit the proliferation, migration, and invasion of CT26 cells and the tube formation of HUVECs in vitro in a dose-dependent manner.

\section{Anlotinib Induces Cell Apoptosis and G2/ $M$ Cell Arrest}

The distribution of cells at different concentrations of anlotinib was analyzed by using flow cytometry to verify whether anlotinib induced cell apoptosis (Figure 2). As anticipated, there was a significant increase in the proportion of cells at the G2/M phase and a decrease in the G0/G1 phase population in the anlotinib-treated CT26 cells. This suggested that anlotinib caused the progression of the cell cycle from the G0/G1 phase to the G2/M phase (Figure $2 \mathrm{~A}$ ). A higher proportion of cells at the $\mathrm{G} 2 / \mathrm{M}$ phase and fewer cells at the G0/G1 phase of the cell cycle were seen at 4 $\mu \mathrm{mol} / \mathrm{L}$ and $8 \mu \mathrm{mol} / \mathrm{L}$ of anlotinib, respectively (Figure $2 \mathrm{~B}$ ). Likewise, the apoptotic rate increased significantly when the concentration of anlotinib was increased (Figure 2C). As illustrated in Figure 2D, the apoptotic rate at the $2 \mu \mathrm{M}$ $(23.56 \pm 2.42 \%), 4 \mu \mathrm{M}(44.98 \pm 10.5 \%)$, and $8 \mu \mathrm{M}(94.07 \pm$ $3.09 \%$ ) was significantly higher as compared with NS group $(9.74 \pm 2.55 \% ; P<0.05)$. Taken together, these data confirmed that anlotinib inhibited the proliferation of CT26 cells through the induction of mitotic arrest and apoptosis.
A
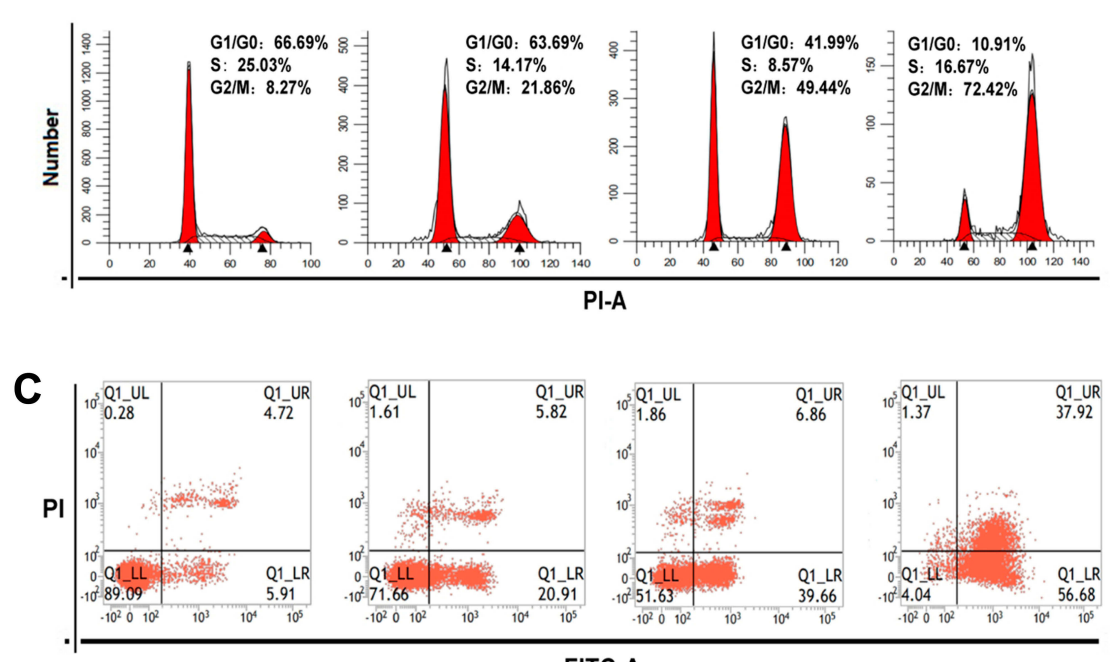

NS

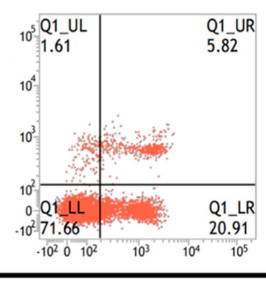

$2 \mu \mathrm{M}$

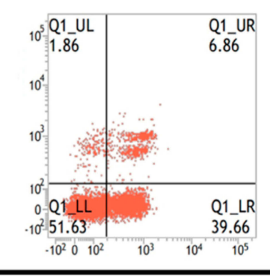

FITC-A

$4 \mu \mathrm{M}$

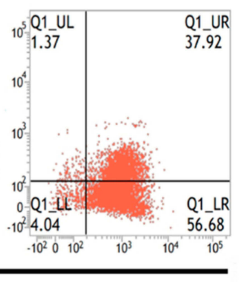

$8 \mu \mathrm{M}$
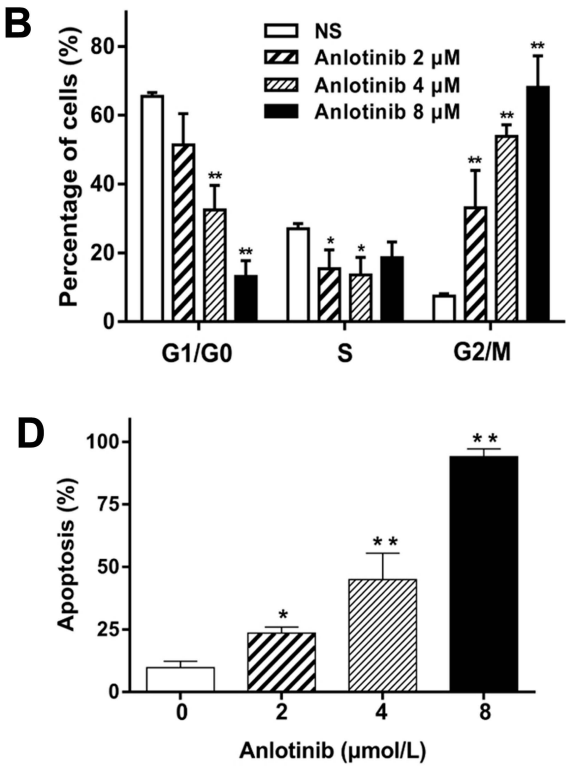

Figure 2 Anlotinib induces G2/M cell cycle arrest and promotes cell apoptosis in CT26 cells. Representative dot plots of the cell cycle after $24 \mathrm{~h}$ of treatment with anlotinib (A) and apoptotic cells after $48 \mathrm{~h}$ of treatment (C) were assessed by flow cytometry. The percentages of the cell cycle (B) and apoptotic cells (D) with different concentrations of Anlotinib. Data were represented as mean \pm SD of three independent experiments. ( $* P<0.05$ and $* * P<0.01$ versus NS group). 
A

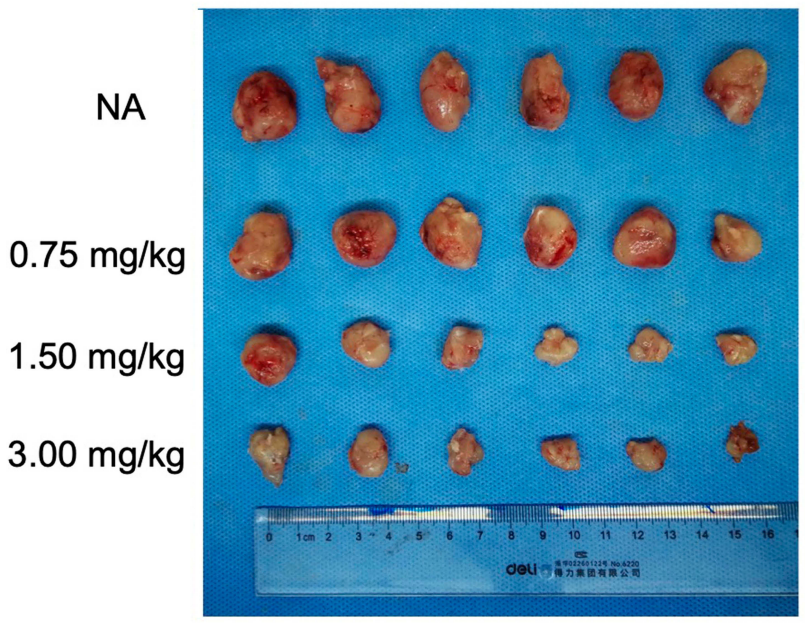

C

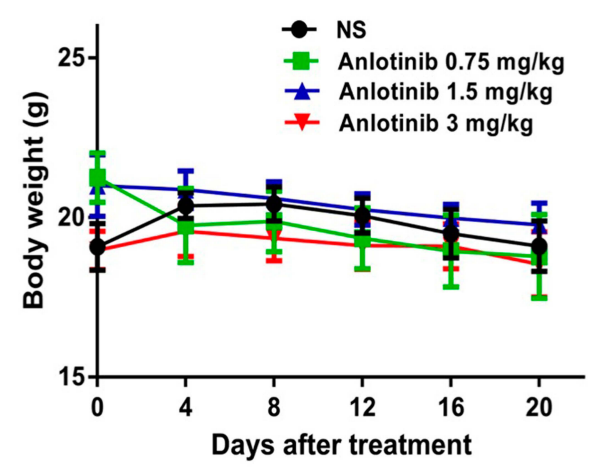

B

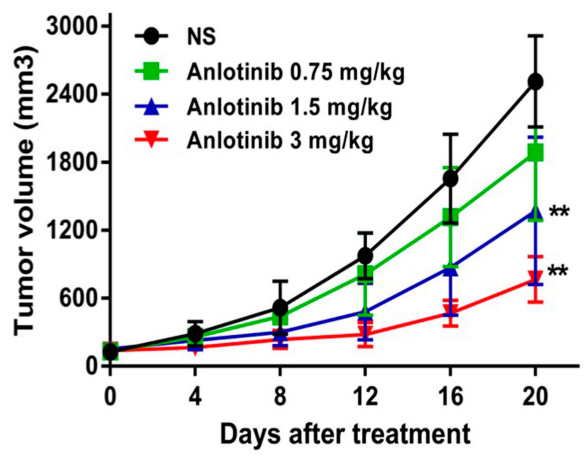

D

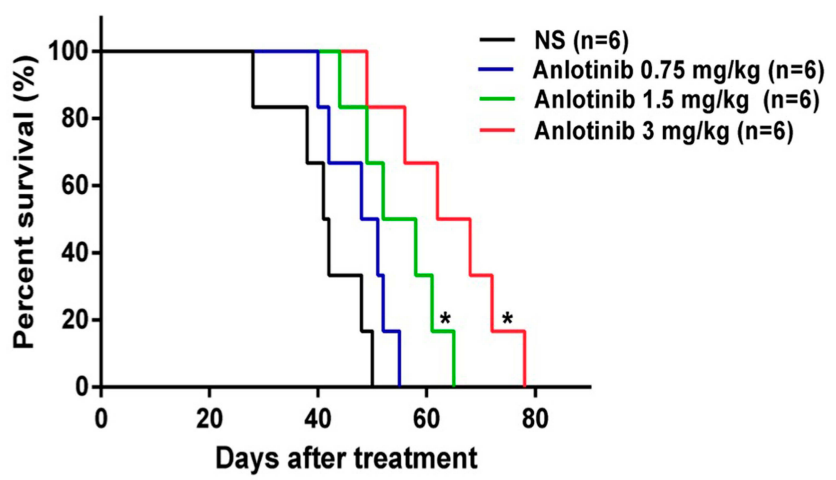

Figure 3 Antineoplastic effect of anlotinib in a xenograft mouse model. (A) Gross image of representative tumors excised after gastric administration of mice with different concentrations of anlotinib. Tumor volume (B) and mouse weight (C) were calculated every 4 days. (D) Survival analysis for the relationship between survival time and different concentrations of anlotinib. Data were represented with a mean \pm SD. The data is representative of three independent experiments. $(* P<0.05$ and $* * P<0.01$ versus NS group).

\section{Anlotinib Suppresses Tumor Growth and Prolongs the Survival Time of Mice in vivo}

The anti-angiogenic effect of anlotinib in vivo was evaluated by using a mouse subcutaneous xenograft model (Figure 3A). After intragastric administration of different concentrations of anlotinib $(0.75,1.5,3 \mathrm{mg} / \mathrm{kg})$, the tumor volume of the $1.5 \mathrm{mg} / \mathrm{kg}$ anlotinib group (1371.25 \pm $649.26 \mathrm{~mm}^{3}$ ) and the $3 \mathrm{mg} / \mathrm{kg}$ anlotinib group $(767.17 \pm$ $\left.200.28 \mathrm{~mm}^{3}\right)$ was significantly lower than NS group $(2513.25 \pm 402.07 \mathrm{~mm} 3)(P<0.01)$. As shown in Figure $3 \mathrm{~B}$, there were no significant differences between the NS group and the $0.75 \mathrm{mg} / \mathrm{kg}$ anlotinib group (2513.25 $\pm 402.07 \mathrm{~mm}^{3}$ vs. $1887.33 \pm 598.35 \mathrm{~mm}^{3} ; P=0.119$ ). Although the different concentrations of anlotinib exhibited a little effect on the bodyweight of mice (Figure 3C), there was no significant difference in the body weight between the four groups $(P>0.05)$. Mice become less active, mentally poor and die with an increase in the tumor load. The median survival time of mice in the $0.75 \mathrm{mg} / \mathrm{kg}, 1.5 \mathrm{mg} / \mathrm{kg}$ and $3 \mathrm{mg} / \mathrm{kg}$ anlotinib group was 48 days, 54 days, and 64 days, respectively while that in the NS group was 41 days (Figure 3D). Moreover, the data revealed that the median survival time of mice treated with anlotinib was significantly prolonged $(P<0.05)$. Collectively, these findings suggested that anlotinib markedly suppressed tumor growth and prolonged the survival time in a mouse xenograft model in vivo.

\section{Anlotinib Inhibits Cell Proliferation and Microvessel Density}

Ki-67+ and CD31+ index of tumor sections were analyzed with immunohistochemistry in order to evaluate cell 
proliferation and microvessel density. Significant differences in expression of $\mathrm{Ki}-67$ and CD31 were seen between each of the three active treatments and the NS group (Figure 4A). As illustrated in Figure 4B, the percentage of Ki-67 positive cells was $32.03 \pm 1.37 \%$ in the $0.75 \mathrm{mg} / \mathrm{kg}$ Anlotinib group, $24.33 \pm 0.74 \%$ in the $1.5 \mathrm{mg} / \mathrm{kg}$ Anlotinib group and $16.74 \pm 1.17 \%$ in the $3 \mathrm{mg} / \mathrm{kg}$ Anlotinib group; which was significantly lower than that in the NS group $(53.13 \pm 3.46 \%$; $P<0.05$ ). Similarly, higher concentrations of anlotinib $(1.5-3 \mathrm{mg} / \mathrm{kg})$ significantly reduced the proportion of CD31 positive expression when compared with the NS group. Notably, a significant reduction of CD31 positive expression was found in $0.75 \mathrm{mg} / \mathrm{kg}$ anlotinib group $(4.5 \pm 0.14 \%)$, $1.5 \mathrm{mg} / \mathrm{kg}$ anlotinib group $(2.42 \pm 0.29 \%)$ and $3 \mathrm{mg} / \mathrm{kg}$ anlotinib group $(1.22 \pm 0.32 \%)$ compared with the NS group $(6.61 \pm 0.16 \%, P<0.05)$ (Figure $4 \mathrm{C})$. Thus, these findings demonstrated that CRC tumor proliferation and angiogenesis in an in vivo mouse xenograft model was suppressed by anlotinib.

\section{Morphological Effect of Anlotinib}

The representative hematoxylin and eosin (H\&E) morphologic findings of anlotinib in the heart, liver, lung, and kidney tissue samples of all the groups have been presented in Figure 5. Histopathological analysis revealed that hepatocytes of groups treated with anlotinib exhibited swelling and balloon-like morphological alterations, which may be attributed to the damaged hepatocytes caused by the drug. However, no apparent metastasis or drug toxicity was seen in vital organs including the heart, lung and kidney.

\section{Anlotinib Inhibits the Activation of VEGFR-2, FGFR and PDGFR $\beta$ and Their Downstream Signaling}

Furthermore, the expressions of VEGFR-2, FGFR, PDGFR $\beta$, AKT, ERK $1 / 2$ and their phosphorylation in mouse xenografts were analyzed by Western blotting to investigate the mechanism through which anlotinib inhibited tumor growth and angiogenesis. The protein expression and phosphorylation levels of all the groups have been presented in Figure 6A. As depicted in Figure 6B, the expression of p-VEGFR2/ VEGFR2 and p-AKT/AKT was significantly decreased in a dose depended manner in the anlotinib group when compared with the NS group $(P<0.05)$. Moreover, a significant decrease in phosphorylation levels of p-FGFR/FGFR and p-PDGFR $\beta /$ PDGFR $\beta(P<0.05)$ was also observed. The expression of $\mathrm{p}$-FGFR/FGFR and $\mathrm{p}$-PDGFR $\beta /$ PDGFR $\beta$ in

A

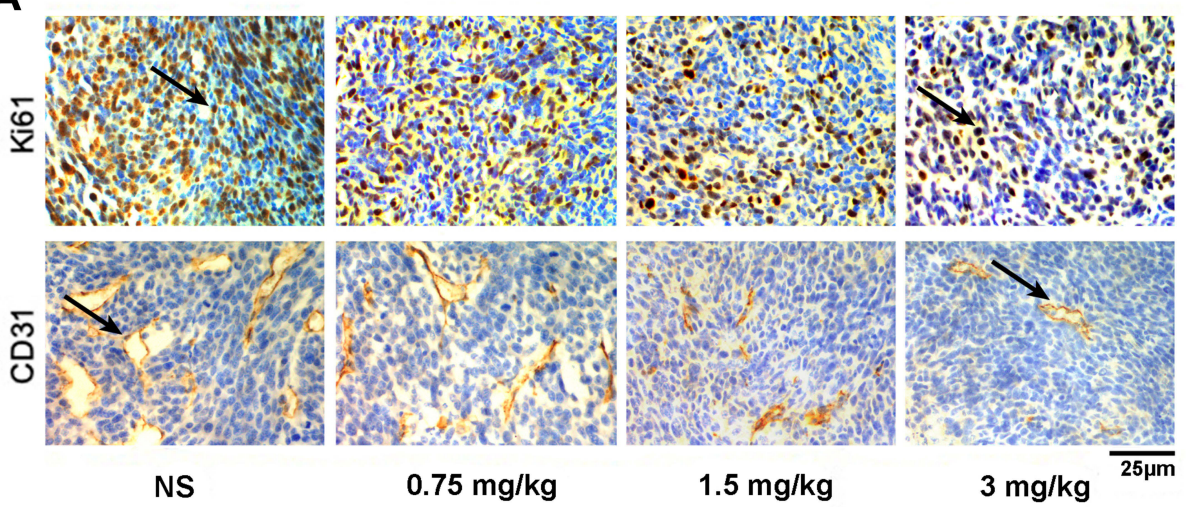

B

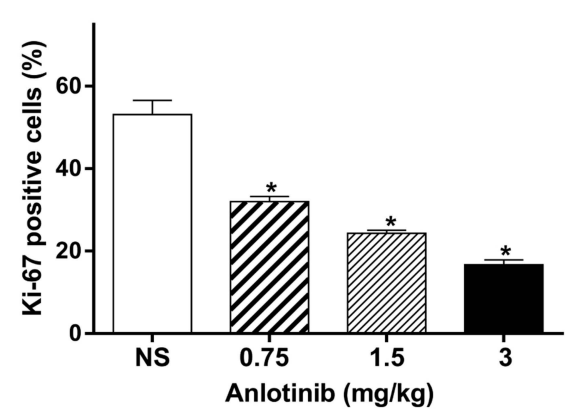

C

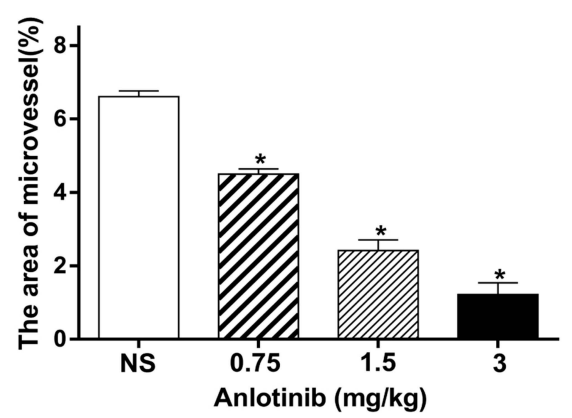

Figure 4 (A) The representative immunohistochemistry staining of $\mathrm{Ki}-67$ and $\mathrm{CD} 3 \mathrm{I}$ in tumor tissues of mice in various groups. (Original magnification $\times 400)$. The black arrow indicates the positive expression. The percentage of positive expression of $\mathrm{Ki}-67(\mathbf{B})$ and $\mathrm{CD} 3 \mathrm{I}(\mathbf{C})$ in various group (*P $<0.05)$. 

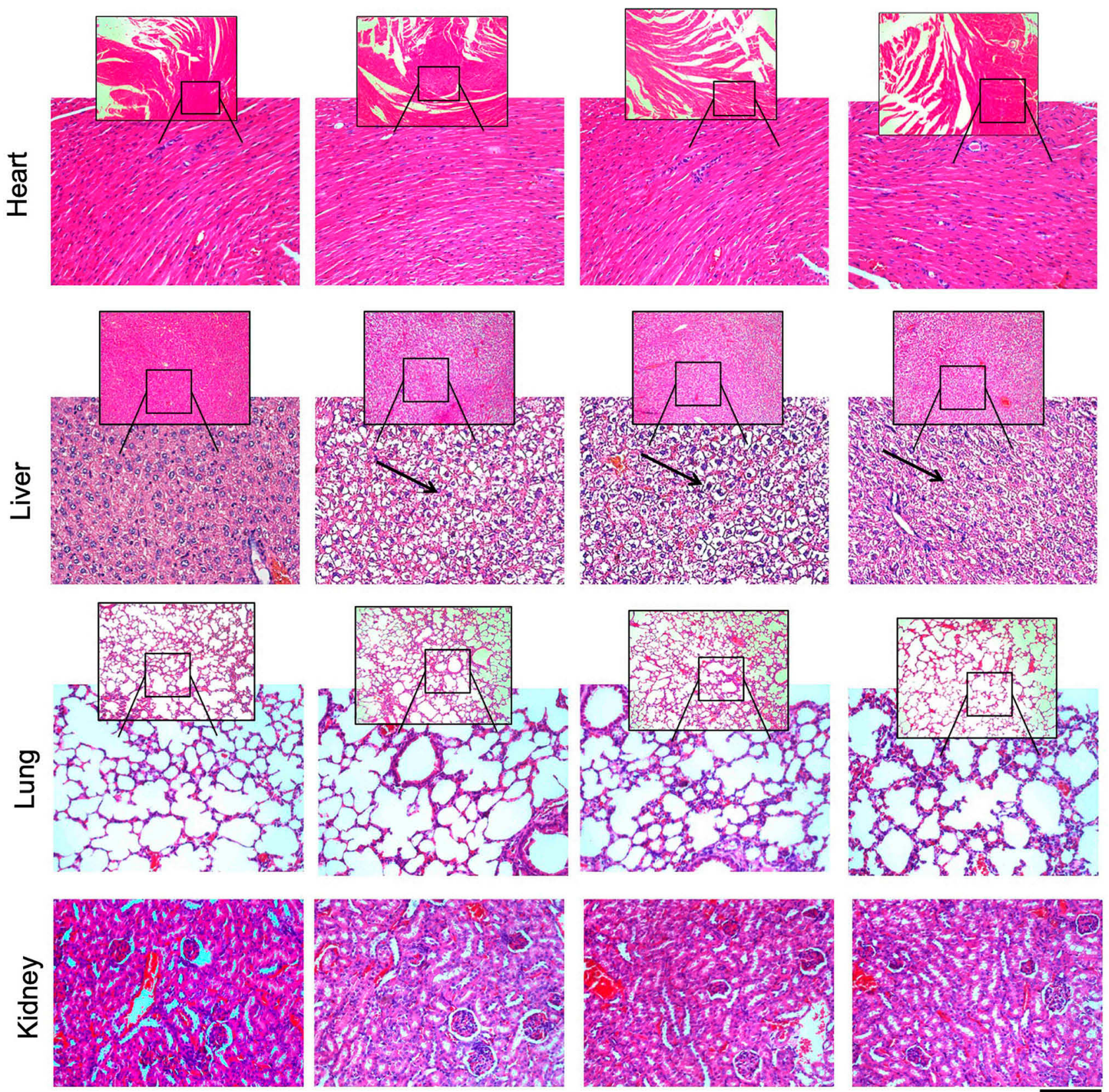

NS

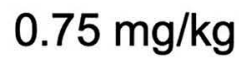

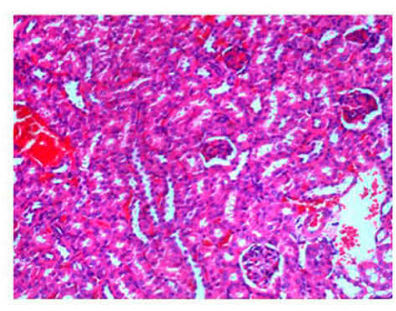

$1.5 \mathrm{mg} / \mathrm{kg}$

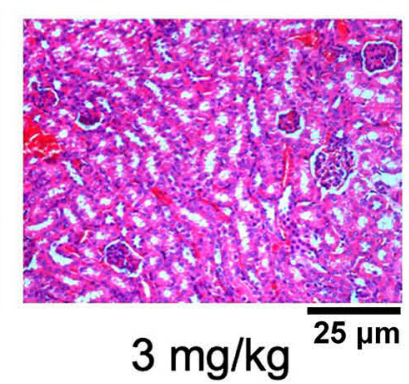

Figure 5 Comparison of representative $\mathrm{H}$ and E-stained heart, liver, lung, and kidney sections between different groups (original magnification: $\times 200$ and $\times 400$ ). The infiltrations of hepatocytes of swelling and balloon-like alterations were observed in the liver (arrow pointed).

the $3 \mathrm{mg} / \mathrm{kg}$ anlotinib group $(0.35 \pm 0.01$ vs.0.67 \pm 0.04 , $\mathrm{p}<0.05)$ and $(0.38 \pm 0.02$ vs. $0.65 \pm 0.03, P<0.05)$ was significantly lower than that in the NS group. Besides, the expression of p-ERK1/2/ERK1/2 in the $3 \mathrm{mg} / \mathrm{kg}$ anlotinib group was significantly lower when compared with the NS group $(0.13 \pm 0.02$ vs. $0.39 \pm 0.02, P<0.05)$. Taken together, these findings indicated that anlotinib inhibited the expression of these proteins and their phosphorylated levels. However, the latter mainly has an inhibitory effect. As a result, anlotinib inhibited the activation of VEGFR-2/AKT in a dose-depended manner and FGFR, PDGFR $\beta$ and their downstream signaling ERK.

\section{Discussion}

Results of our study have demonstrated that anlotinib could inhibit proliferation, migration, invasion and angiogenesis, regulate the cell cycle, and induce apoptosis in CT26 cells via down-regulation of multiple targets and the AKT/ERK 
A

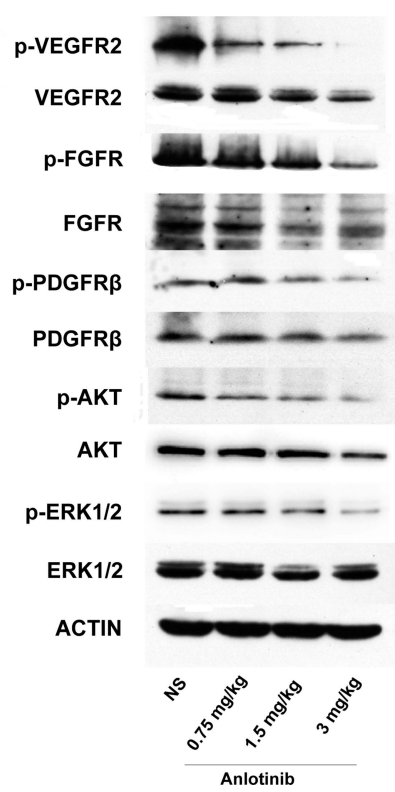

B

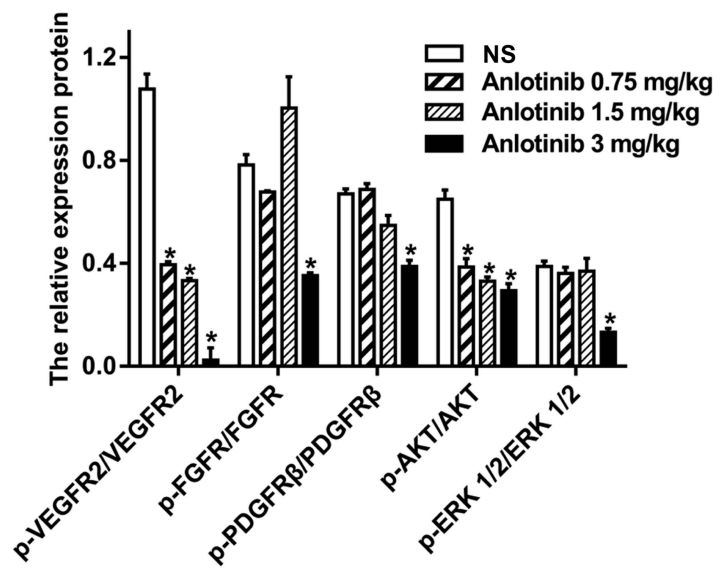

Figure 6 Anlotinib suppresses AKT/ERK signaling cascade via blocking VEGFR-2, FGFR, and PDGFR $\beta$. (A) Representative Western blot analysis of P-VEGFR2, VEGFR2, p-FGFR, FGFR, p-PDGFR $\beta$, PDGFR $\beta$, p-AKT, AKT, p-ERKI/2, and ERKI/2 protein in various groups. (B) The relative expression of p-VEGFR2/VEGFR2, p-FGFR/FGFR, P-PDGFR $\beta / P D G F R \beta, p-A K T / A K T$ and $\mathrm{p}$-ERKI/2/ERKI/2 in various groups. In Western blot, ACTIN was used as an internal control (*P $<0.05$ versus $N S$ group).

signaling cascade. Our study also dissected the probable molecular mechanisms through which the anti-tumor effects of anlotinib are mediated.

Proliferation and metastasis of tumor cells are the major biological characteristics of CRC tumor formation. ${ }^{26,27}$ Thus, the efficacy of antitumor agents always depends on their ability to cause inhibition of tumor proliferation, migration, and invasion. In-vitro results from our study demonstrated that anlotinib remarkably suppressed proliferation, migration and invasion of CT26 cells in a dose-dependent manner. Expansion of tumors is promoted by neovascularization in the tumors which provides oxygen and nutrients for tumor growth. ${ }^{14,15}$ Angiogenesis is an extremely complex process which involves interconnection of vascular endothelial cells for formation of new vascular structures, which is one of the key links in angiogenesis. ${ }^{28}$ In the current study, anlotinib caused potent inhibition of microvessel outgrowth in endothelial cells can destroyed the formation of vascular structures at low concentrations. As disruption of the cell cycle is one of the hallmarks of cancer, thus this process represents the most crucial target of therapeutic drugs. ${ }^{29}$ Moreover, several previous studies have demonstrated that anlotinib promoted cell apoptosis and cell cycle arrest in a variety of cancers including lung cancer, ${ }^{8}$ liver cancer, ${ }^{9}$ osteosarcoma ${ }^{10}$ and thyroid cancer. ${ }^{11}$ As anticipated, anlotinib caused the arrest of the cell cycle at the G2/M phase and promoted cell apoptosis of CT26 cells. Besides, our study revealed that anlotinib-treated mice had significantly smaller tumor volume than the control group. The median survival time was also significantly prolonged in the anlotinib-treated mice when compared with the control group. Furthermore, histopathological analysis of the heart, liver, lung, and kidney tissues from the xenograft mouse model revealed that anlotinib caused significant hepatocyte damage. This suggests that the monitoring of liver function is essential for cancer patients receiving anlotinib. Accumulating studies indicated that the downregulation of Ki-67 and CD31 in tumor tissues have confirmed the inhibitory effects of anlotinib on the proliferation of tumor cells and microvessels. ${ }^{8,10,30}$ Therefore, the expression of Ki-67 and CD31 was detected by IHC and our results in a mouse xenograft model suggested that anlotinib down-regulated biomarkers of microvessel density (CD31) and proliferation (Ki-67). These results were consistent with the in-vitro results which provided evidence on the anti-proliferative and antiangiogenic effects of anlotinib on CT26 cells.

Tumor angiogenesis has been attributed to an imbalance between pro-angiogenic and anti-angiogenic factors as well as increases tumor growth. ${ }^{31}$ Therefore, inhibition of angiogenesis is an attractive strategy for treatment of cancer. ${ }^{32}$ VEGF and its receptor, VEGFR2 play a critical role in tumor angiogenesis. Binding of VEGF to VEGFR2 is 
responsible for the phosphorylation of VEGFR2 and the activation of AKT and ERK1/2 which are the downstream signaling molecules in the VEGF pathway. AKT and ERK1/ 2 promote endothelial cell growth, migration, and survival. $^{25,33}$ Similar to VEGF, FGF-2 triggers the autophosphorylation of FGFR1 and activates downstream signaling cascades and angiogenesis. ${ }^{34}$ Besides, PDGF-BB binds to its receptor PDGFR $\beta$ to regulate tumor angiogenesis, growth and metastasis. ${ }^{35}$ Results from our study demonstrated that anlotinib potently inhibited the activation of VEGFR2, FGFR, and PDGFR $\beta$ and their downstream signaling molecules AKT and ERK1/2.

Most of the studies conducted till dates have only examined the use of anlotinib in cancer treatment as a monotherapy. Certainly, these data need to be substantiated by an appropriate perspective and comprehensive study. Based on the findings of the current study, we created a molecular model which demonstrated the anti-tumorigenic effect of anlotinib on the CT26 cells. The aforementioned results will be helpful to dissect out the mechanisms via which anlotinib inhibits CRC progression. Further studies on anlotinib in CRC are warranted to study whether the combination of anlotinib and other therapies result in an enhanced anti-tumor effect. The long term toxicity of anlotinib in CRC also needs to be analyzed further.

In conclusion, the findings of the present study revealed that anlotinib exhibits a strong inhibitory effect on the growth, metastasis, and angiogenesis of CRC through attenuation of VEGFR2, FGFR, and PDGFR $\beta$ signaling pathways both in vitro and in vivo. Besides, this study also provided prospective evidence for the clinical application of anlotinib in the therapeutic management of mCRC.

\section{Acknowledgments}

The authors thank people who provided technical assistant and financial support in Oncology Department, First Affiliated Hospital of Southwest Medical University. We also thankfully acknowledge all authors for their continuous collaborations with us in sharing information and contributions.

\section{Funding}

This work was supported by grants from Southwest Medical University and the Research Foundation of the Science and Technology Department of Sichuan Province [No. 0903-00021897].

\section{Disclosure}

No potential conflicts of interest were disclosed.

\section{References}

1. Sepulveda AR, Hamilton SR, Allegra CJ, et al. Molecular biomarkers for the evaluation of colorectal cancer: guideline from the American Society for Clinical Pathology, College of American Pathologists, Association for Molecular Pathology, and the American Society of Clinical Oncology. J Clin Oncol. 2017;35:1453-1486. doi:10.1200/ JCO.2016.71.9807

2. Global Burden of Disease Cancer Collaboration; Fitzmaurice C, Abate D, et al. Global, regional, and national cancer incidence, mortality, years of life lost, years lived with disability, and disability-adjusted life-years for 29 Cancer Groups, 1990 to 2017: a systematic analysis for the Global Burden of Disease Study. JAMA Oncol. 2019;5(12):1749-1768.

3. Elferink MAG, de Jong KP, Klaase JM, et al. Metachronous metastases from colorectal cancer: a population-based study in North-East Netherlands. Int J Colorectal Dis. 2015;30(2):205-212. doi:10.1007/ s00384-014-2085-6

4. Riihimäki M, Hemminki A, Sundquist J, et al. Patterns of metastasis in colon and rectal cancer. Sci Rep. 2016;6(1):29765. doi:10.1038/ srep29765

5. Kirstein MM, Lange A, Prenzler A, et al. Targeted therapies in metastatic colorectal cancer: a systematic review and assessment of currently available data. Oncologist. 2014;19(11):1156-1168. doi:10.1634/theoncologist.2014-0032

6. Siegel RL, Miller KD, Jemal A. Cancer statistics, 2018. CA Cancer J Clin. 2018;68(1):7-30. doi:10.3322/caac.21442

7. Shen G, Zheng F, Ren D, et al. Anlotinib: a novel multi-targeting tyrosine kinase inhibitor in clinical development. J Hematol Oncol. 2018;11(1):120. doi:10.1186/s13045-018-0664-7

8. Xie C, Wan X, Quan H, et al. Preclinical characterization of anlotinib, a highly potent and selective vascular endothelial growth factor receptor-2 inhibitor. Cancer Sci. 2018;109:1207-1219. doi:10.1111/ cas. 13536

9. Liang L, Hui K, $\mathrm{Hu} \mathrm{C}$, et al. Autophagy inhibition potentiates the anti-angiogenic property of multikinase inhibitor anlotinib through JAK2/STAT3/VEGFA signaling in non-small cell lung cancer cells. J Exp Clin Cancer Res. 2019;38:71. doi:10.1186/s13046-019-1093-3

10. He C, Wu T, Hao Y. Anlotinib induces hepatocellular carcinoma apoptosis and inhibits proliferation via Erk and Akt pathway. Biochem Biophys Res Commun. 2018;503:3093-3099. doi:10.1016/ j.bbrc.2018.08.098

11. Wang G, Sun M, Jiang Y, et al. Anlotinib, a novel small molecular tyrosine kinase inhibitor, suppresses growth and metastasis via dual blockade of VEGFR2 and MET in osteosarcoma. Int $J$ Cancer. 2019;145:979-993. doi:10.1002/ijc.32180

12. Ruan X, Shi X, Dong Q, et al. Antitumor effects of anlotinib in thyroid cancer. Endocr Relat Cancer. 2019;26:153-164. doi:10.1530/ ERC-17-0558

13. Taurin S, Yang $\mathrm{CH}$, Reyes $\mathrm{M}$, et al. Endometrial cancers harboring mutated fibroblast growth factor receptor 2 protein are successfully treated with a new small tyrosine kinase inhibitor in an orthotopic mouse model. Int J Gynecol Cancer. 2018;28:152-160. doi:10.1097/ IGC.0000000000001129

14. Jayson GC, Kerbel R, Ellis LM, et al. Antiangiogenic therapy in oncology: current status and future directions. Lancet. 2016;388 (10043):518-529. doi:10.1016/S0140-6736(15)01088-0

15. El-Kenawi AE, El-Remessy AB. Angiogenesis inhibitors in cancer therapy: mechanistic perspective on classification and treatment rationales. $B r \quad J$ Pharmacol. 2013;170:712-729. doi:10.1111/ bph.12344 
16. Shweiki D, Itin A, Soffer D, et al. Vascular endothelial growth factor induced by hypoxia may mediate hypoxia-initiated angiogenesis. Nature. 1992;359(6398):843-845. doi:10.1038/359843a0

17. Kim KJ, Li B, Winer J, et al. Inhibition of vascular endothelial growth factor-induced angiogenesis suppresses tumour growth in vivo. Nature. 1993;362(6423):841-844. doi:10.1038/362841a0

18. Ferrara N, Gerber H-P, LeCouter J. The biology of VEGF and its receptors. Nat Med. 2003;9(6):669-676. doi:10.1038/nm0603-669

19. Lin B, Song X, Yang D, et al. Anlotinib inhibits angiogenesis via suppressing the activation of VEGFR2, PDGFR $\beta$ and FGFR1. Gene. 2018;654:77-86. doi:10.1016/j.gene.2018.02.026

20. Han B, Li K, Zhao Y, et al. Anlotinib as a third-line therapy in patients with refractory advanced non-small-cell lung cancer: a multicentre, randomised phase II trial (ALTER0302). $\mathrm{Br}$ $J$ Cancer. 2018;118:654-661. doi:10.1038/bjc.2017.478

21. Han B, Li K, Wang Q, et al. Effect of anlotinib as a third-line or further treatment on overall survival of patients with advanced non-small cell lung cancer: the ALTER 0303 phase 3 randomized clinical trial. JAMA Oncol. 2018;4:1569-1575. doi:10.1001/jamaoncol.2018.3039

22. Sun Y, Du F, Gao M, et al. Anlotinib for the treatment of patients with locally advanced or metastatic medullary thyroid cancer. Thyroid. 2018;28:1455-1461. doi:10.1089/thy.2018.0022

23. Xie F, Ding RL, He WF, et al. In vivo antitumor effect of endostatin-loaded chitosan nanoparticles combined with paclitaxel on Lewis lung carcinoma. Drug Deliv. 2017;24(1):1410-1418. doi:10.1080/10717544.2017.1378938

24. Liu Z-J-L, Zhou Y-J, Ding R-L, et al. In vitro and in vivo apatinib inhibits vasculogenic mimicry in melanoma MUM-2B cells. PLoS One. 2018;13(7):e0200845. doi:10.1371/journal.pone.0200845

25. Olsson AK, Dimberg A, Kreuger J, et al. VEGF receptor signalling - in control of vascular function. Nat Rev Mol Cell Biol. 2006;7:359-371. doi:10.1038/nrm1911
26. Ren QG, Huang T, Yang SL, et al. Colon cancer metastasis to the mandibular gingiva with partial occult squamous differentiation: a case report and literature review. Mol Clin Oncol. 2017;6:189-192. doi:10.3892/mco.2016.1102

27. Mandel JS, Bond JH, Church TR, et al. Reducing mortality from colorectal cancer by screening for fecal occult blood. Minnesota Colon Cancer Control Study. N Engl J Med. 1993;328:1365-1371. doi:10.1056/NEJM199305133281901

28. Carmeliet P, Jain RK. Molecular mechanisms and clinical applications of angiogenesis. Nature. 2011;473(7347):298-307. doi:10.1038/ nature 10144

29. Stark GR, Taylor WR. Analyzing the G2/M checkpoint. Methods Mol Biol. 2004;280:51-82. doi:10.1385/1-59259-788-2:051

30. Li HY, Yang HG, Li P, et al. Effect of heat treatment on the antitumor activity of lactoferrin in human colon tumor (HT29) model. J Agric Food Chem. 2019;67:140-147. doi:10.1021/acs.jafc.8b05131

31. Viallard C, Larrivée B. Tumor angiogenesis and vascular normalization: alternative therapeutic targets. Angiogenesis. 2017;20:409-426. doi:10.1007/s10456-017-9562-9

32. Paduch R. The role of lymphangiogenesis and angiogenesis in tumor metastasis. Cell Oncol (Dordr). 2016;39:397-410. doi:10.1007/ s13402-016-0281-9

33. Hicklin DJ, Ellis LM. Role of the vascular endothelial growth factor pathway in tumor growth and angiogenesis. $J$ Clin Oncol. 2005;23:1011-1027. doi:10.1200/JCO.2005.06.081

34. Katoh M, Nakagama H. FGF receptors: cancer biology and therapeutics. Med Res Rev. 2014;34:280-300. doi:10.1002/ med.21288

35. Zhao Y, Adjei AA. Targeting angiogenesis in cancer therapy: moving beyond vascular endothelial growth factor. Oncologist. 2015;20:660-673. doi:10.1634/theoncologist.2014-0465

\section{Publish your work in this journal}

Cancer Management and Research is an international, peer-reviewed open access journal focusing on cancer research and the optimal use of preventative and integrated treatment interventions to achieve improved outcomes, enhanced survival and quality of life for the cancer patient.
The manuscript management system is completely online and includes a very quick and fair peer-review system, which is all easy to use. Visit http://www.dovepress.com/testimonials.php to read real quotes from published authors. 